\title{
MicroRNA-92a promotes non-small cell lung cancer cell growth by targeting tumor suppressor gene FBXW7
}

\author{
DA NI, JIPING TENG, YOUSHUANG CHENG, ZHIJUN ZHU, BUFENG ZHUANG and ZHIYIN YANG \\ Department of Thoracic Surgery, Shanghai Ninth People's Hospital, \\ Shanghai JiaoTong University School of Medicine, Shanghai 201999, P.R. China
}

Received June 5, 2019; Accepted April 15, 2020

DOI: $10.3892 / \mathrm{mmr} .2020 .11373$

\begin{abstract}
MicroRNA (miRNA/miR)-92a has been identified as being significantly downregulated in non-small cell lung cancer (NSCLC) tissues using a miRNA array. However, its biological function and molecular mechanisms in NSCLC have not been fully elucidated. The aim of the present study was to determine the role of miR-92a in NSCLC and the mechanisms by which it affects NSCLC cells. The expression levels of miR-92a in NSCLC tissues and cell lines were analyzed using reverse transcription-quantitative PCR. Cell viability and cell apoptosis were determined using an MTT assay and flow cytometry, respectively. It was observed that miR-92a was significantly upregulated in NSCLC tissues and cell lines. Inhibition of miR-92a significantly suppressed viability of NSCLC cells, with concomitant downregulation of key proliferative genes, such as proliferating cell nuclear antigen and Ki-67. miR-92a downregulation induced apoptosis of NSCLC cells, as evidenced by flow cytometry and apoptosis-related protein detection. Luciferase assays confirmed that miR-92a could directly bind to the 3'-untranslated region of tumor suppressor F-box/WD repeat-containing protein 7 (FBXW7) and suppress its translation. Furthermore, small interfering RNA-mediated FBXW7 inhibition partially attenuated the tumor suppressive effect of an miR-92a inhibitor on NSCLC cells. Collectively, these findings demonstrated that miR-92a might function as an oncogene in NSCLC by regulating FBXW7. In conclusion, miR-92a could serve as a potential therapeutic target in NSCLC treatment.
\end{abstract}

\section{Introduction}

Lung cancer is one of the leading causes of cancer-related mortality in China (1). Among the two major lung cancer

Correspondence to: Dr Zhiyin Yang, Department of Thoracic Surgery, Shanghai Ninth People's Hospital, Shanghai JiaoTong University School of Medicine, 280 Mohe Road, Baoshan, Shanghai 201999, P.R. China

E-mail: zhiyinyang@126.com

Key words: microRNA-92a, FBXW7, non-small cell lung cancer subtypes, non-small-cell lung cancer (NSCLC) accounts for $\geq 80 \%$ of all lung cancer cases (2). Over the past several years, surgical resection has remained the main choice of treatment for patients with NSCLC, but the survival rate of these patients remains $\leq 18 \%$ due to rapid tumor metastasis $(3,4)$. Therefore, there is a pressing need to identify new highly sensitive biomarkers and targets for therapeutic intervention to aid in the diagnosis of NSCLC and improve patient outcomes.

MicroRNAs (miRNAs/miRs) are single-stranded non-coding RNAs that negatively regulate gene expression by binding to the 3'-untranslated region (UTR) of their target gene mRNAs at the post-transcriptional level (5). Previous studies have revealed that miRNAs are crucial for various biological and pathological processes, and aberrant expression or function of miRNAs are commonly observed in various types of cancer, including NSCLC (6-8). It has been shown that the overexpression of miR-30e could inhibit cell viability and invasion by targeting SRY-box transcription factor 9 in NSCLC cells (9). Liu et al (10) also revealed that miR-661 promoted NSCLC viability, migration and metastasis by targeting retinoblastoma 1 . However, the role of miRNAs in NSCLC and the molecular mechanisms by which they affect this type of cancer remain largely unknown.

miR-92a, a putative oncogene, is frequently upregulated in numerous types of human cancer (11). For example, Yu et al (12) demonstrated that upregulation of miR-92a promoted the viability, migration and invasion of osteosarcoma cells. Shigoka et al (13) reported that miR-92a was upregulated in hepatocellular carcinoma ( $\mathrm{HCC}$ ) and overexpression of miR-92a enhanced HCC cell viability. A recent study also confirmed the oncogenic role of miR-92a in colorectal cancer (CRC), and revealed its pro-proliferative and anti-apoptotic functions in CRC cells (14). Jiang et al (15) demonstrated that overexpression of miR-92a promoted osteosarcoma tumor growth in vivo. In a previous study, miR-92a was more highly expressed in NSCLC tumor tissues than in adjacent tissue samples and plasma from healthy donors (16). However, the mechanisms by which miR-92a affects NSCLC tumorigenesis remains unclear. Therefore, the aim of the present study was to examine the potential molecular mechanisms by which miR-92a affects the pathogenesis of NSCLC cells by examining miR-92a expression in NSCLC tissues and cell lines. The regulatory role of miR-92a in NSCLC cell viability 
and apoptosis, and the relevant mechanisms by which it affects NSCLC cells, were also investigated.

\section{Materials and methods}

Patients and sample collection. The present study was approved by the Research Ethics Committee of Shanghai Ninth People's Hospital, Shanghai JiaoTong University School of Medicine (Shanghai, China). All patients provided written, informed consent. Paired NSCLC tissues and adjacent normal tissues were obtained from 15 patients ( 9 men, 6 women; age, $60.3 \pm 8.5$ years) during surgery between January and June 2018. None of the patients with NSCLC received treatment before surgery. In addition, SCLC tissues and adjacent normal tissue were collected from 15 patients $(8 \mathrm{men}, 7$ women; age, 62.1 \pm 9.2 years) before chemotherapy between August and November 2019. All samples were collected at the Department of Thoracic Surgery, Shanghai Ninth People's Hospital, Shanghai JiaoTong University School of Medicine, and both tumor and adjacent normal tissues were confirmed by pathological examinations. Patients were excluded if they had recurrent tumors or had primary tumors but received chemoradiotherapy before surgical operation. Tissues were immediately snap frozen in liquid nitrogen and stored at $-80^{\circ} \mathrm{C}$ prior to RNA extraction.

Cell lines and culture conditions. The four NSCLC cell lines (A549, H358, NCI-H520 and H1299), a normal human bronchial epithelial cell line (16HBE) and 293T cells were purchased from The Cell Bank of Type Culture Collection of the Chinese Academy of Sciences. All cells were cultured in DMEM (Gibco; Thermo Fisher Scientific, Inc.) supplemented with $10 \%$ fetal bovine serum (Invitrogen; Thermo Fisher Scientific, Inc.), $100 \mathrm{U} / \mathrm{ml}$ penicillin and $100 \mathrm{mg} / \mathrm{ml}$ streptomycin, in humidified air at $37^{\circ} \mathrm{C}$ with $5 \% \mathrm{CO}_{2}$.

$R N A$ extraction and reverse transcription-quantitative $P C R$ $(R T-q P C R)$. Total RNA was extracted from NSCLC tissues and cell lines with TRIzol ${ }^{\circledR}$ reagent (Invitrogen; Thermo Fisher Scientific, Inc.), according to the manufacturer's instructions. For RT-qPCR, RNA was reverse transcribed to cDNA from 100 ng total RNA using a RT kit (Tiangen Biotech Co., Ltd.), and qPCR was performed with SYBRGreen (Tiangen Biotech Co., Ltd.). All protocols were carried out according to the manufacturer's instructions. The primer sequences are listed as follows: miR-92a, forward 5'-CCGCGTGCTGGGATTC-3', reverse, 5'-TCCAGAAGGCTGCAAATGG-3'; U6, forward 5'-CTCGCTTCGGCAGCACA-3', reverse 5'-GTCATACTC CTGCTTGCTGAT-3'; F-box/WD repeat-containing protein 7 (FBXW7), forward 5'-GTCCCGAGA AGCGGTTTG ATA-3', reverse 5'-TGCTCAGGCACGTCAGAAAAG-3'; and GAPDH, forward 5'-GAAGATGGTGATGGGATTTC-3', and reverse 5'-AACGCTTCACGAATTTGCGT-3'. RT-qPCR was performed on a Step-One Plus Real-Time PCR system (Applied Biosystems; Thermo Fisher Scientific, Inc.), and each RT-qPCR reaction was performed in triplicate, including no-template controls. The reaction was performed under the following conditions: $95^{\circ} \mathrm{C}$ for $5 \mathrm{~min}$, followed by 40 cycles at $95^{\circ} \mathrm{C}$ for $15 \mathrm{sec}$ and $60^{\circ} \mathrm{C}$ for $50 \mathrm{sec}$, and a final extension at $72^{\circ} \mathrm{C}$ for $10 \mathrm{~min}$. The relative quantification of miR-92a and FBXW7 were normalized to the expression of U6 and GAPDH, respectively using the $2^{-\Delta \Delta \mathrm{Cq}}$ method (17).

Cell transfection. The miR-92a mimics (5'-UAUUGCACU UGUCCCGGCCUGU-3'), mimics negative control (NC; 5'-CGGTGUGUUCAG ACUACC UGUUC-3'), miR-92a inhibitor (5'-ACAGGCCGGGACAAGUGCAAUA-3') and inhibitor NC (5'-TAACACGTCTATACGCCCA-3') were obtained from Guangzhou RiboBio Co., Ltd. FBXW7 small interfering (si)RNA (si-FBXW7; sense, 5'-TAAAGAGTT GGCACTCTAT-3' and antisense, 5'-ATAGAGTGCCAACTC TTTA-3') and corresponding NC siRNA (si-scramble; sense, 5'-TTCTCCGAACGTGTCACGT-3' and antisense, 5'-ACG TGACACGTTCGGAGA A-3') were also purchased from Guangzhou RiboBio Co., Ltd.

Upon A549, H1299, H358 and NCI-H520 cells in six-well plates reaching $80 \%$ confluence, $1 \times 10^{6}$ cells/well were transfected with the transfectants using Lipofectamine ${ }^{\circledR} 2000$ reagent (Invitrogen; Thermo Fisher Scientific, Inc.), according to the manufacturer's protocol. A final concentration of $50 \mathrm{nM}$ miR-92a mimics, $100 \mathrm{nM}$ mimics $\mathrm{NC}, 200 \mathrm{nM}$ miR-92a inhibitor, $100 \mathrm{nM}$ inhibitor NC, $100 \mathrm{nM}$ si-FBXW7 or $100 \mathrm{nM}$ si-Scramble were used for each transfection. A blank control (untransfected cells) was set up for each transfection. Following transfection at $37^{\circ} \mathrm{C}$ for $24 \mathrm{~h}$, the transfection efficiency was analyzed using RT-qPCR or western blotting.

Cell viability. The effect of miR-92a on NSCLC cell viability was measured using an MTT assay. After transfection with the indicated miRNA mimics, inhibitor or siRNA, $20 \mu \mathrm{l}$ MTT solution (Sigma-Aldrich; Merck KGaA) was added to each well (2x10 /well), and A549, H1299, H358 and NCI-H520 cells were cultured for an additional $2 \mathrm{~h}$ at 24,36 and $48 \mathrm{~h}$ post-transfection. Then, $150 \mu 1$ dimethyl sulfoxide was used to dissolve the purple formazan. Subsequently, the absorbance of the samples at $450 \mathrm{~nm}$ was detected using a microplate reader (Bio-Rad Laboratories, Inc.).

Flow cytometry. At 24-h post-transfection, apoptotic cells were detected using Annexin V/APC and propidium iodide (PI) apoptosis detection kit I (BD Pharmingen; BD Biosciences), according to the manufacturer's protocol. Briefly, NSCLC cells were collected after centrifugation at $400 \mathrm{x} \mathrm{g}$ for $10 \mathrm{~min}$ at room temperature, and then washed with cold PBS. Then, $1 \times 10^{5}$ cells $/ \mathrm{ml}$ were stained with binding buffer, containing $5 \mu \mathrm{l}$ Annexin V-FITC and $10 \mu 1 \mathrm{PI}$ at $4^{\circ} \mathrm{C}$ in the dark for $15 \mathrm{~min}$. Following the incubation, cell apoptosis was analyzed using a FACScan flow cytometer (BD Biosciences) and CellQuest software version 3.3 (BD Biosciences).

Luciferase reporter assay. The targets of miR-92a were predicted using TargetScan 7.0 (http://www.targetscan.org) and miRanda (http://www.microrna.org). The 3'-untranslated region (UTR) fragment of FBXW7 containing the putative wild-type (wt) sequence was amplified by PCR. The amplified product was inserted into the pGL3 luciferase reporter vector (Promega Corporation), namely pGL-FBXW7-wt. The QuikChange Lightning Site-Directed Mutagenesis kit (Stratagene; Agilent Technologies, Inc.) was used to construct the miR-92a binding site mutation in the 3'-UTR 
of FBXW7, according to the manufacturer's protocol; this construct was named pGL-FBXW7-mutant (mut). A total of $2 \times 10^{5} 293 \mathrm{~T}$ cells/well were seeded into 24 -well plates and transfected with miR-92a mimics or mimics $\mathrm{NC}$, and 100 ng pGL-FBXW7-wt or pGL-FBXW7-mut, together with $100 \mathrm{ng}$ pRL-TK Renilla plasmids (Promega Corporation) using Lipofectamine ${ }^{\circledR} 2000$ (Invitrogen; Thermo Fisher Scientific, Inc.) at $37^{\circ} \mathrm{C}$. A total of $48 \mathrm{~h}$ after transfection, the luciferase activity was determined using a Dual-Luciferase Reporter Assay system (Promega Corporation), according to the manufacturer's protocol. Relative luciferase activity was normalized to Renilla luciferase activity.

Western blot analysis. Total protein was extracted from cells using RIPA buffer (Sigma-Aldrich; Merck KGaA) with $1 \%$ phenylmethylsulfonyl fluoride. The protein concentration was determined using a BCA protein assay kit (Pierce; Thermo Fisher Scientific, Inc.). Total protein samples $(30 \mu \mathrm{g})$ were separated by SDS-PAGE on $8 \%$ gels and transferred to a polyvinylidene fluoride membrane (EMD Millipore). The membranes were blocked with $5 \%$ non-fat milk at $4^{\circ} \mathrm{C}$ overnight and incubated with primary antibodies overnight at $4^{\circ} \mathrm{C}$. Primary antibodies against Bax (1:1,000; cat. no. sc-70408), Bcl-2 (1:1,000; cat. no. sc-7382), proliferating cell nuclear antigen (PCNA; 1:1,000; cat. no. sc-9857) and $\beta$-actin (1:2,000; cat. no. sc-8432) were purchased from Santa Cruz Biotechnology, Inc., while cleaved caspase-3 (1:1,000; cat. no. 9661) and Ki-67 (1:1,000; cat. no. 12075) were purchased from Cell Signaling Technology, Inc. After washing with PBS, the membrane was incubated with HRP-conjugated antibodies (1:2,000; cat. nos. ab205718 and ab97040; Abcam) for $1 \mathrm{~h}$ at room temperature and the bands were detected with an ECL Advance reagent (GE Healthcare). The intensity of the bands of interest was analyzed with ImageJ software version 1.46 (National Institutes of Health).

Statistical analyses. SPSS 13.0 software (SPSS, Inc.) was used to analyze the data. Data were expressed as the mean \pm SD of three independent experiments. Differences between two groups were analyzed by a paired t-test when comparing paired NSCLC tissues and adjacent normal tissues, and an unpaired t-test for comparing two groups in other cases. Differences between multiple groups were analyzed by one-way analysis of variance, followed by Tukey's post-hoc test. Pearson's correlation analysis was carried out to determine the association between miR-92a and FBXW7. P<0.05 was considered to indicate a statistically significant difference.

\section{Results}

miR-92a is upregulated in NSCLC tissues and cell lines. To investigate the potential involvement of miR-92a in NSCLC development, RT-qPCR analysis was conducted to determine miR-92a expression levels in 15 paired NSCLC and adjacent tissues. As shown in Fig. 1A, the expression levels of miR-92a were significantly upregulated in NSCLC tissues compared with in adjacent tissues. In addition, higher expression levels of miR-92a were also observed in SCLC tissues compared with adjacent tissues (Fig. 1B), which is consistent with the results of a previous study (18). This analysis was also extended to four NSCLC cell lines; A549, H1299, NCI-H520 and H358, with $16 \mathrm{HBE}$ as a control. Consistent with the results of miR-92a expression levels in clinical tissues, miR-92a expression was markedly increased in these cell lines compared with in 16HBE cells (Fig. 1C). Together, these data suggested that miR-92a may be involved in NSCLC progression.

Inhibition of miR-92 a suppresses the viability of NSCLC cells. According to the aforementioned detection of miR-92a expression in the four NSCLC cell lines, the highly metastatic A549 and H1299 cell lines, in which miR-92a was highly expressed, were chosen to conduct loss-of-function experiments. miRNA transfection efficiency was evaluated using RT-qPCR. As shown in Fig. 2A, miR-92a was significantly decreased in A549 and H1299 cells post-transfection with the miR-92a inhibitor. The biological effects of miR-92a inhibition on regulating cell viability was then assessed using an MTT assay. Inhibition of miR-92a significantly suppressed A549 and H1299 cell viability compared with inhibitor NC-transfected cells (Fig. 2B). PCNA and Ki-67 expression may reflect the degree of viability because their rate of synthesis is directly associated with the rates of cellular proliferation and DNA synthesis (19). Therefore, to further confirm the miR-92a inhibitor-induced reduction in cell viability, the protein expression levels of PCNA and Ki-67 were examined. Western blot analysis indicated that miR-92a inhibition resulted in a significant decrease in PCNA and Ki-67 protein expression (Fig. 2C). Therefore, these results demonstrated that inhibition of miR-92a could suppress the viability of NSCLC cells.

Inhibition of miR-92a promotes apoptosis of NSCLC cells. To determine whether apoptosis inhibited growth, the effects of miR-92a inhibitor on cell apoptosis were assessed. It was demonstrated that miR-92a inhibition markedly promoted apoptosis of A549 and H1299 cells compared in the inhibitor NC group (Fig. 3A and B). In addition, miR-92a inhibition significantly increased the expression levels of pro-apoptotic proteins, cleaved caspase- 3 and Bax, and reduced the expression levels of the anti-apoptotic protein Bcl-2 (Fig. 3C and D). These data suggested that miR-92a inhibition promoted apoptosis of NSCLC cells.

Overexpression of miR-92a inhibits the viability and induces cell apoptosis of NSCLC cells. Next, weakly metastatic cells H358 and NCI-H520, which expressed lower levels of miR-92a compared with the other NSCLC cell lines, were used for gain-of-function experiments. As shown in Fig. 4A, miR-92a was significantly increased in H358 and NCI-H520 cells post-transfection with miR-92a mimics. The MTT assay revealed that miR-92a overexpression significantly promoted cell viability, compared with that in mimics NC-transfected cells (Fig. 4B). Apoptosis of H358 and NCI-H520 cells was also markedly suppressed with miR-92a overexpression (Fig. 4C). Collectively, miR-92a overexpression may promote cell viability and suppress cell apoptosis in vitro.

miR-92a directly targets $F B X W 7$. To explore the molecular mechanism by which miR-92a mediates its inhibitory effect on NSCLC, TargetScan 7.0 and miRanda analyses were conducted to predict the target genes of miR-92a. FBXW7, 

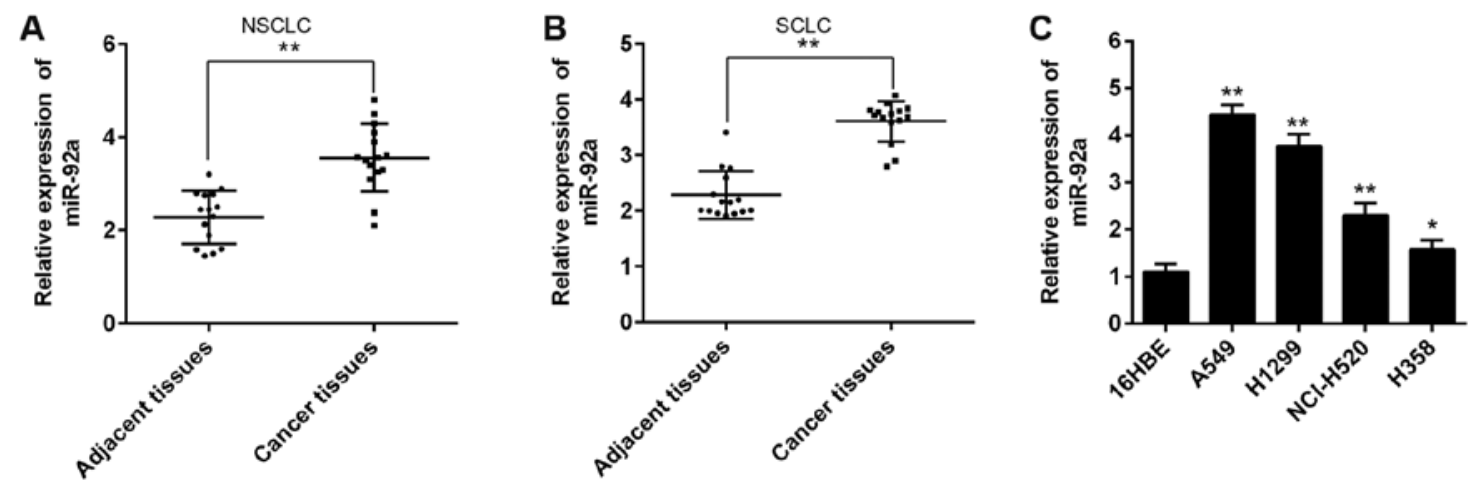

Figure 1. Expression levels of miR-92a in NSCLC tissues and cell lines. (A) miR-92a expression was examined by RT-qPCR in 15 paired NSCLC tissues and adjacent tissues. Paired Student's $t$ test; ${ }^{* * *} \mathrm{P}<0.01$ vs. adjacent tissues. (B) Expression of miR-92a was determined by RT-qPCR in SCLC tissues. Paired Student's $\mathrm{t}$ test; ${ }^{* *} \mathrm{P}<0.01$ vs. adjacent tissues. (C) Expression of miR-92a was determined by RT-qPCR in A549, H1299, NCI-H520 and H358 NSCLC cell lines. A normal human bronchial epithelial cell line, $16 \mathrm{HBE}$, was used as a control. Data are presented as mean of three replicates \pm SD and analyzed using one-way ANOVA with a Tukey's post hoc test; ${ }^{*} \mathrm{P}<0.05,{ }^{* *} \mathrm{P}<0.01$ vs. 16HBE. NSCLC, non-small-cell lung cancer; miR, microRNA; RT-qPCR, reverse transcription-quantitative PCR; SCLC, small-cell lung cancer.

A

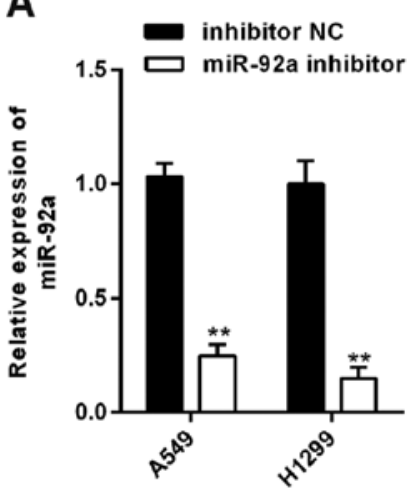

C

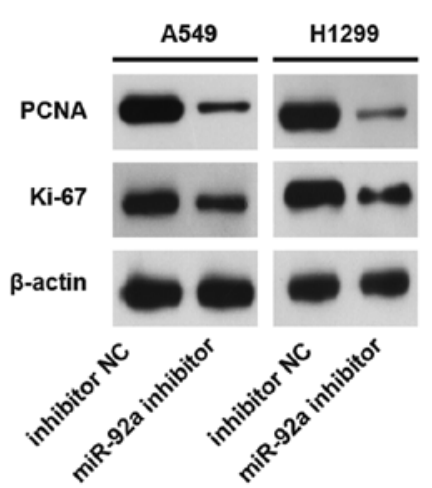

B
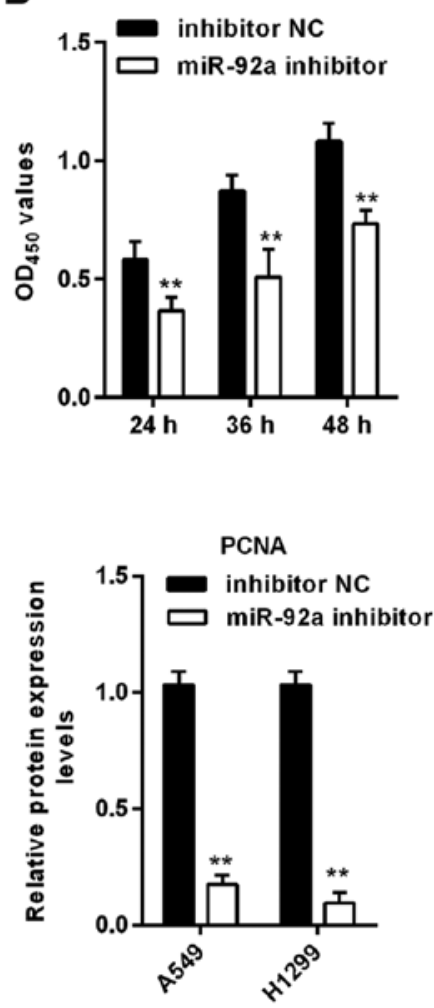

H1299
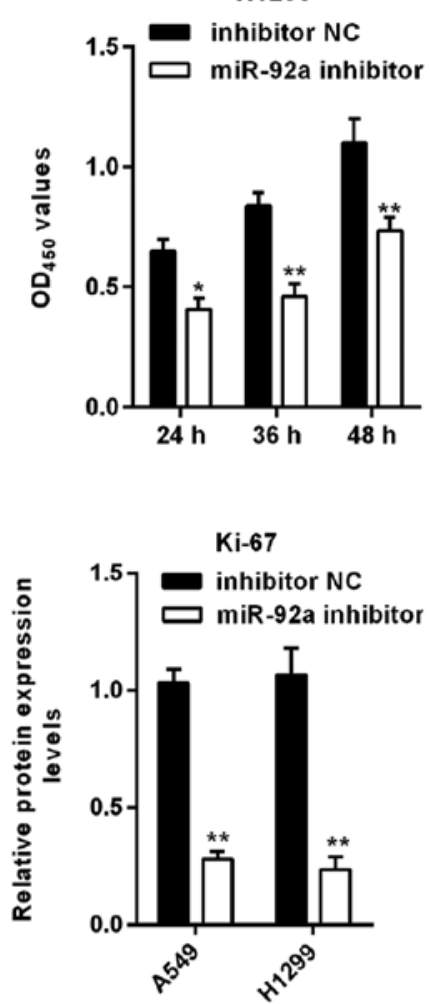

Figure 2. Inhibition of miR-92a suppresses viability of non-small-cell lung cancer cells. (A) miR-92a expression was measured by reverse transcription-quantitative PCR after miR-92a inhibitor transfection. (B) miR-92a inhibitor was transfected into A549 and H1299 cells. The effects of miR-92a on A549 and H1299 cell viability were examined using an MTT assay at 24, 36 and 48 h post-transfection. (C) Protein expression levels of PCNA and Ki-67 in A549 and H1299 cells were measured by western blot analysis. Relative protein expression levels of PCNA and Ki-67 were normalized to the protein expression levels of $\beta$-actin. Data are presented as mean of three replicates $\pm \mathrm{SD}$. Unpaired t-test; ${ }^{*} \mathrm{P}<0.05$ and ${ }^{* *} \mathrm{P}<0.01$ vs. inhibitor $\mathrm{NC}$ group. NC, negative control; miR, microRNA; PCNA, proliferating cell nuclear antigen.

a well-known tumor suppressor, was identified as a potential target of miR-92a, with the target site located in the 3'-UTR of FBXW7 mRNA (Fig. 5A). To validate whether FBXW7 is a direct target gene of miR-92a, the 3'-UTR region of FBXW7 was fused to a luciferase system. As shown in Fig. 5B, miR-92a mimics significantly suppressed the luciferase activities of the
3'-UTR segment of FBXW7, but not those of the construct containing a mut binding site (mut FBXW7 3'-UTR), compared with the NC group.

Western blotting was conducted to further determine whether miR-92a regulated the expression of FBXW7. The results revealed that FBXW7 expression levels were markedly 
A

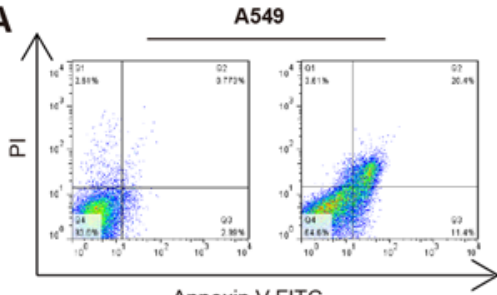

inhibitor NC
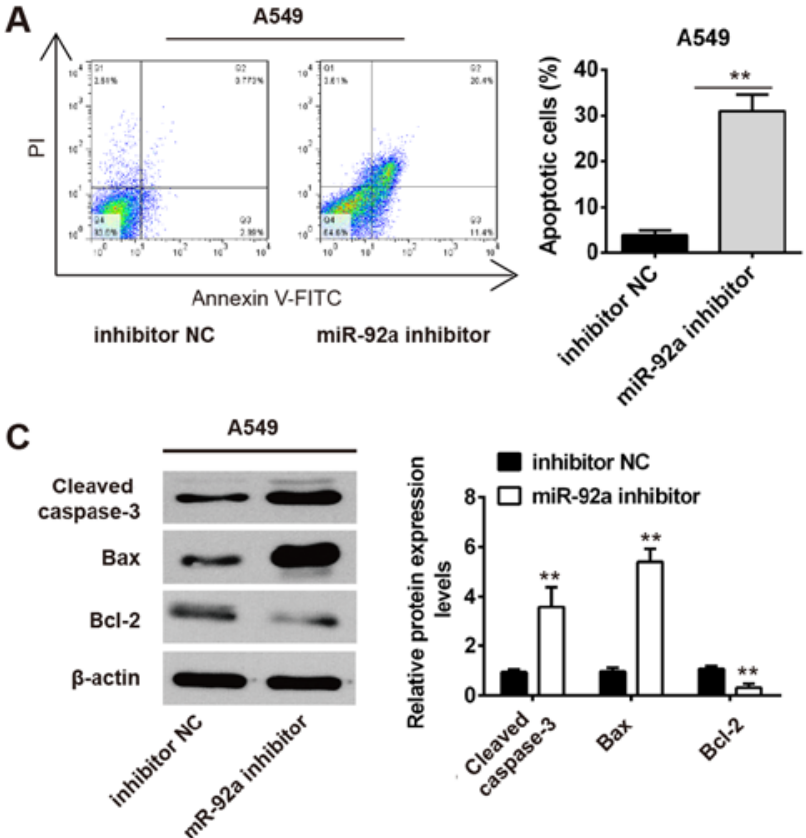

B

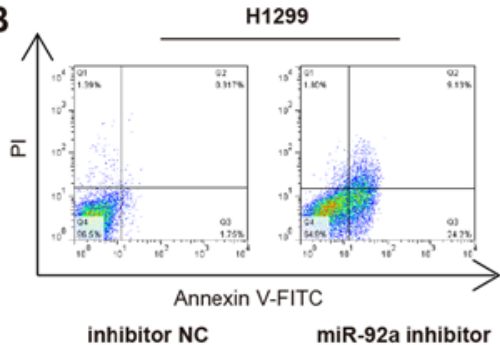

inhibitor NC

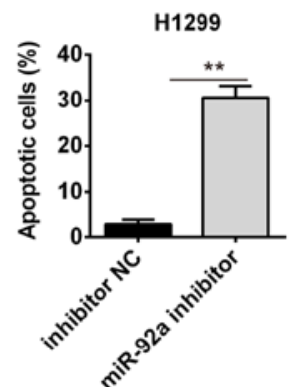

D

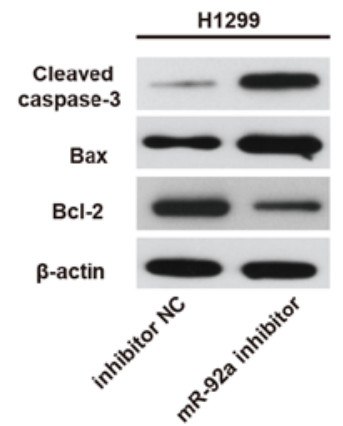

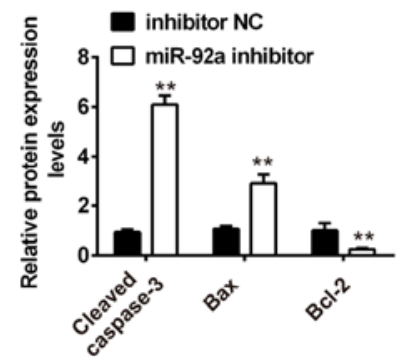

Figure 3. Inhibition of miR-92a promotes apoptosis of non-small-cell lung cancer cells. (A) A549 and (B) H1299 cells were transfected with miR-92a inhibitor for $24 \mathrm{~h}$. Then, the effects of miR-92a inhibition on A549 and H1299 cell apoptosis were examined using flow cytometry. Protein expression levels of cleaved caspase-3, Bcl-2 and Bax in (C) A549 and (D) H1299 cells were measured by western blot analysis. Data are presented as mean of three replicates \pm SD. Unpaired t-test; ${ }^{* *} \mathrm{P}<0.01$ vs. inhibitor NC group. NC, negative control; miR, microRNA; PI, propidium iodide.

A

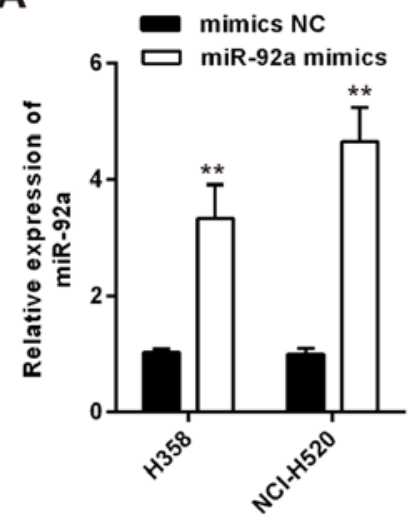

B

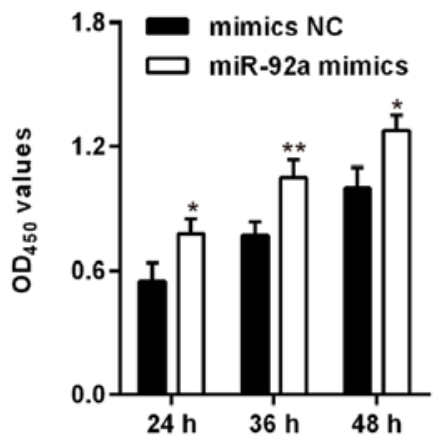

$\mathrm{NCl}-\mathrm{H} 520$

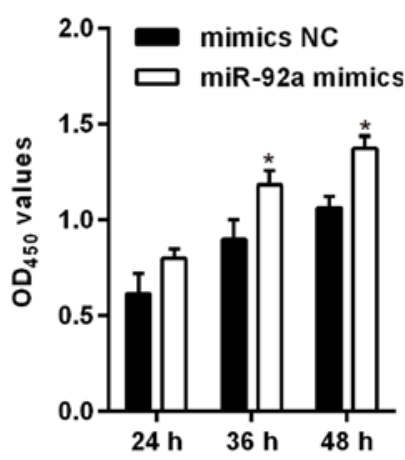

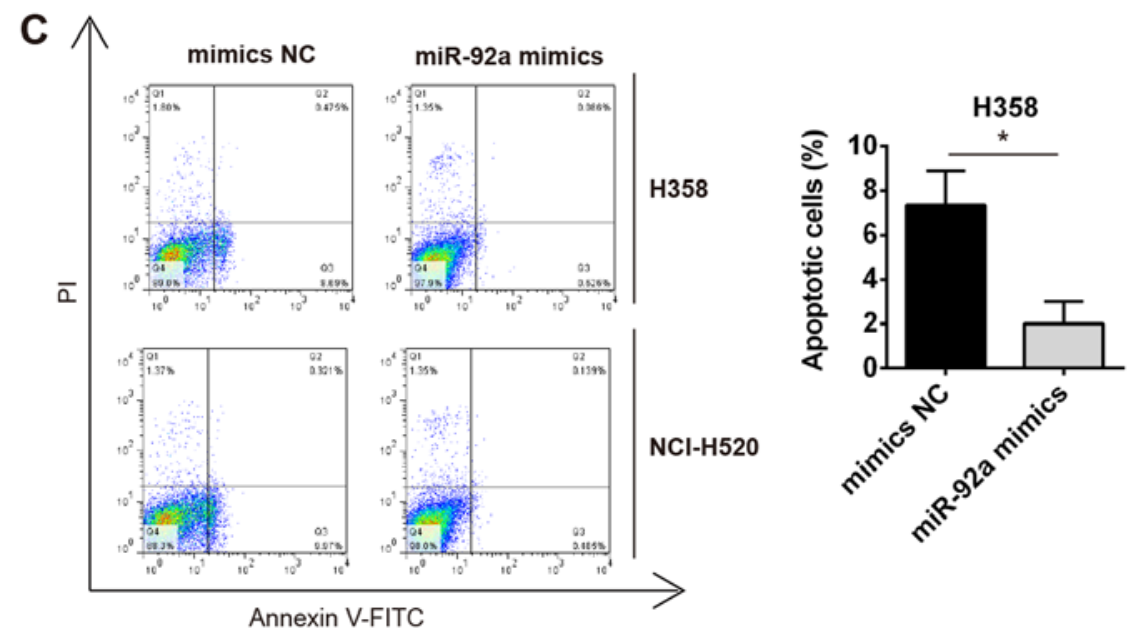

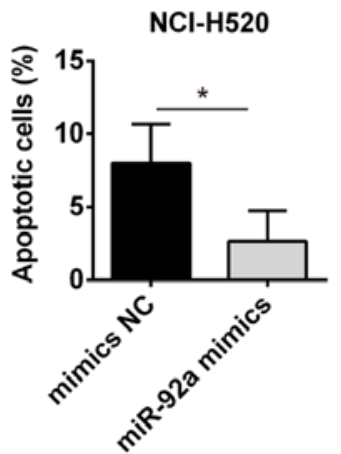

Figure 4. Overexpression of miR-92a promotes proliferation and inhibits apoptosis of non-small-cell lung cancer cells. (A) Expression of miR-92a was measured by reverse transcription-quantitative PCR after miR-92a mimics transfection. (B) miR-92a mimics were transfected into NCI-H520 and H358 cells. Then, the effects of miR-92a on cell viability were examined using an MTT assay at 24,36 and $48 \mathrm{~h}$ post-transfection. (C) The effects of miR-92a mimics on cell apoptosis were examined using flow cytometry. Data are presented as mean of three replicates $\pm \mathrm{SD}$. Unpaired $\mathrm{t}$-test; ${ }^{*} \mathrm{P}<0.05,{ }^{* * *} \mathrm{P}<0.01$ vs. mimics $\mathrm{NC}$ group. NC, negative control; miR, microRNA; PI, propidium iodide. 
A

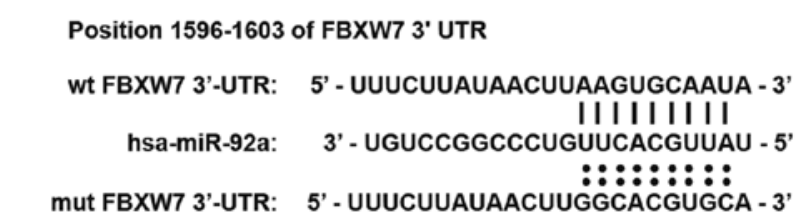

B

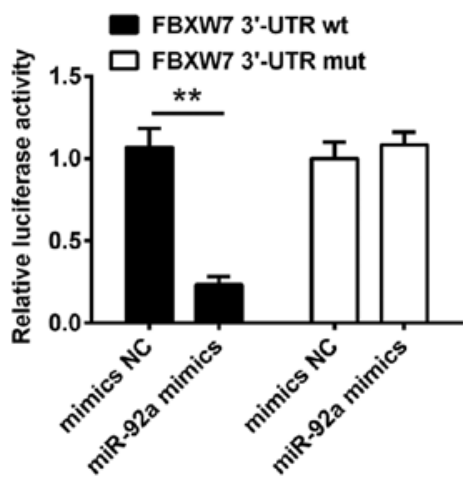

E

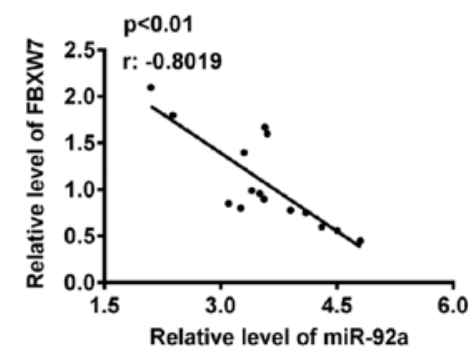

Figure 5. FBXW7 is a direct target of miR-92a. (A) Prediction of miR-92a binding site in FBXW7 3'-UTR. (B) Luciferase assay of 293 T co-transfected with firefly luciferase constructs containing the FBXW7 wt or mut 3'-UTR and miR-92a mimics or mimics NC. Data are presented as the mean of three replicates \pm SD. ${ }^{* *} \mathrm{P}<0.01$ vs. mimics NC. (C) Protein expression of FBXW7 after transfection with miR-92a mimics in H358 cells, as measured by western blotting. (D) Levels of FBXW7 mRNA expression were determined by reverse transcription-quantitative PCR in 15 paired non-small-cell lung cancer and adjacent tissues. Paired t-test; ${ }^{* *} \mathrm{P}<0.01$ vs. adjacent tissues. (E) The relationship between miR-92a and FBXW7 expression was assessed by Spearman correlation analysis; r=-0.8019, P<0.01. UTR, untranslated region; miR, microRNA; FBXW7, F-box/WD repeat-containing protein 7; wt, wild type; mut, mutant.

downregulated by the miR-92a mimics (Fig. 5C). The mRNA expression levels of FBXW7 were detected in 15 pairs of NSCLC and adjacent tissues using RT-qPCR. The results revealed that FBXW7 mRNA expression was significantly downregulated in NSCLC tissues compared with in adjacent tissues (Fig. 5D). Further analyses indicated that FBXW7 expression was negatively correlated with miR-92a expression in NSCLC tissues (Fig. 5E). These data suggested that miR-92a directly targets FBXW7 and suppresses its expression in NSCLC cells.

Downregulation of miR-92a inhibits cell viability and promotes apoptosis by targeting $F B X W 7$. As aforementioned, FBXW7 was a direct target of miR-92a in NSCLC cells; therefore, it was further investigated whether downregulation of miR-92a exerted its antiproliferative and pro-apoptotic effects by upregulating FBXW7. A549 and H1299 cells were co-transfected with siRNA-FBXW7 and miR-92a inhibitor, and cell viability and apoptosis were evaluated. Firstly, siRNA-FBXW7 transfection efficiency was confirmed by western blotting. The results revealed that siRNA-FBXW7 transfection markedly inhibited FBXW7 levels compared with siRNA-scramble transfection in A549 cells and H1299 cells (Fig. 6A). Compared with untransfected cells, miR-92a inhibitor significantly reduced cell viability, whereas this inhibitory effect was attenuated by siRNA-mediated FBXW7 knockdown (Fig. 6B and C). Meanwhile, siRNA-FBXW7 could significantly weaken apoptosis enhancement caused by miR-92a inhibitor in A549 and H1299 cells (Fig. 6D and E). Collectively, these data suggested that miR-92a downregulation may suppress NSCLC cell growth and enhance apoptosis by targeting FBXW7.

\section{Discussion}

The present study demonstrated that miR-92a was upregulated in NSCLC tissues and cell lines. Moreover, miR-92a downregulation suppressed cell viability and promoted cell apoptosis in vitro by targeting the tumor suppressor FBXW7. These findings suggested that miR-92a may potentially provide a novel strategy for the treatment of NSCLC.

Emerging evidence has indicated that aberrant expression of miRNAs can affect tumorigenesis processes by regulating key oncogenes or tumor suppressors (20); therefore, miRNAs may have great potential as prognostic indicators and therapeutic targets (21). Previous studies have demonstrated that aberrant miRNA expression may contribute to NSCLC progression (22,23). For example, Yang et al (24) showed that miR-218 was significantly downregulated in lung cancer tissues and was associated with prognosis of patients with lung cancer. Zhuang et al (25) reported that serum levels of miR-484 could help screen patients for NSCLC with a high sensitivity and specificity using receiver operating curve analyses. In the present study, miR-92a was significantly upregulated in NSCLC tissues and cell lines, which suggested that miR-92a could potentially act as a new diagnostic marker and therapeutic target in NSCLC treatment.

Previous studies have also examined the oncogenic role of miR-92a in other types of human cancer $(14,26,27)$. For 

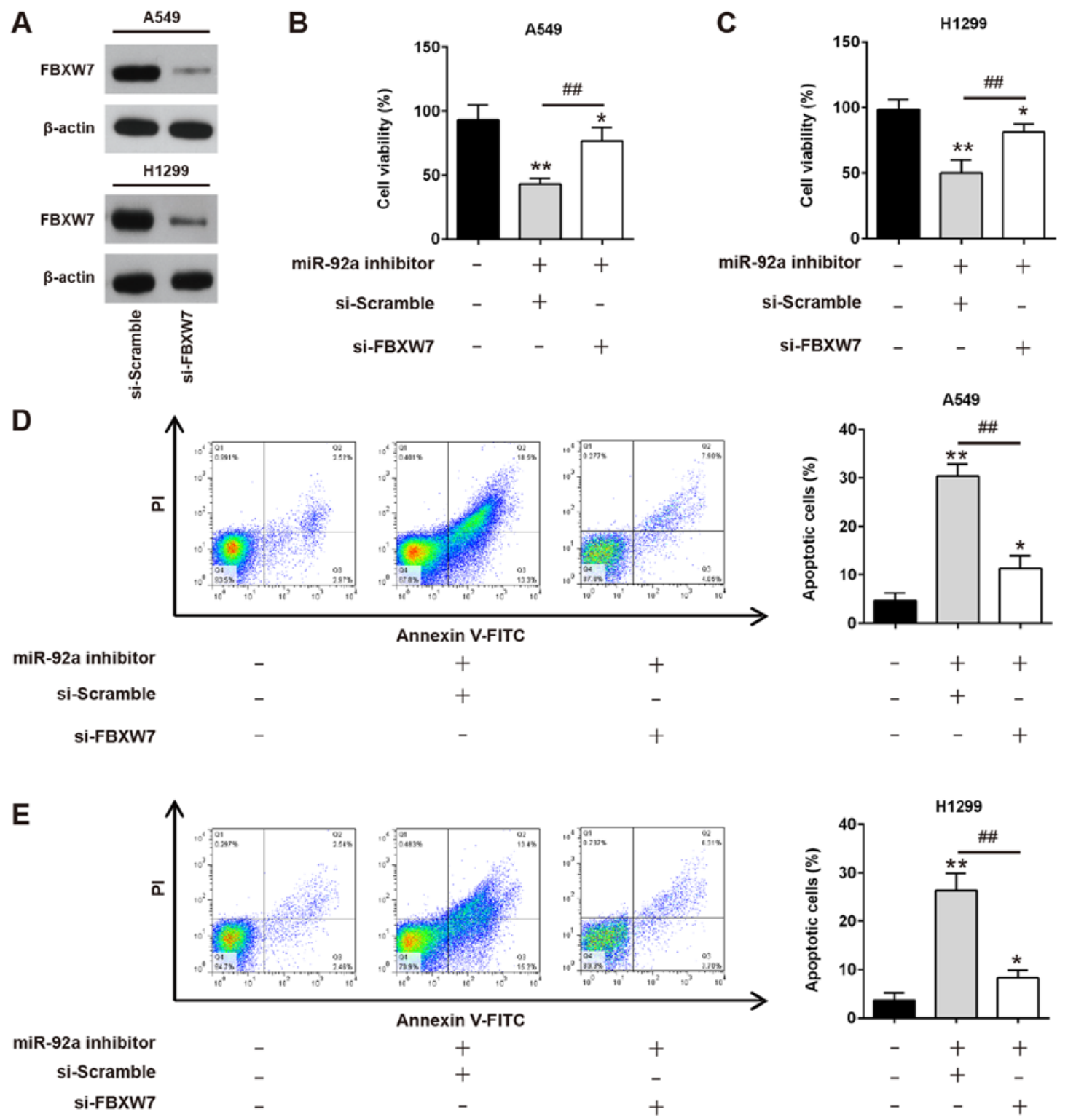

Figure 6. Downregulation of miR-92a inhibits cell viability and promotes apoptosis by targeting FBXW7. A549 and H1299 cells were co-transfected with si-FBXW7 (or si-Scramble) and miR-92a inhibitor, and cell viability and apoptosis were evaluated. Non-transfected cells were used as a control (Blank group). (A) Transfection efficiency of si-FBXW7 was confirmed by western blotting. (B and C) Cell viability was measured by an MTT assay. (D and E) Apoptosis was determined by flow cytometry. Data are presented as mean of three replicates \pm SD. One-way ANOVA with Tukey's post hoc test; ${ }^{*} \mathrm{P}<0.05,{ }^{* *} \mathrm{P}<0.01$ vs. blank group; ${ }^{\# \#} \mathrm{P}<0.01$ vs. miR-92a inhibitor group. FBXW7, F-box/WD repeat-containing protein 7; miR, microRNA; si, small interfering; PI, propidium iodide.

example, Ke et al (28) showed that miR-92a promoted CRC cell metastasis through the PTEN-mediated PI3K/AKT pathway. Chen et al (29) demonstrated that miR-92a promoted the migration and invasion of human esophageal squamous cell carcinoma by, at least partially, suppressing cadherin-1 expression. Notably, miR-92a has been observed to be highly expressed in NSCLC tissues and cell lines $(30,31)$, which indicates that miR-92a may be involved in NSCLC progression. $\mathrm{Lu}$ et al (32) revealed that miR-92a regulated cell migration and invasion of NSCLC cells by targeting PTEN; therefore, it was hypothesized that miR-92a may affect NSCLC progression, which had not been previously investigated. The results of the present study demonstrated that miR-92a inhibition could suppress cancer cell viability (with suppressed PCNA and Ki-67 protein expression) and promote apoptosis (with increased caspase- 3 and Bax, and decreased Bcl-2 protein expression). Together, these results suggested that miR-92a may function as an oncogene in NSCLC progression.

To elucidate the mechanisms by which NSCLC progression is induced by miR-92a, bioinformatics analyses were conducted to predict the putative targets of miR-92a, and FBXW7 was identified as one of these potential targets. FBXW7 has been reported to be a tumor suppressor in human tumorigenesis, with marked effects on cell cycle progression, cell growth and invasion $(33,34)$. For example, Li et al (35) revealed that upregulation of FBXW7 attenuated osteosarcoma cell proliferation and its low expression was associated with a worse patient outcome. Xiao et al (36) also demonstrated that FBXW7 knockdown enhanced NSCLC tumorigenesis and resistance to gefitinib in a xenograft mouse model. Notably, a previous study (15) showed that overexpression of miR-92a promoted osteosarcoma growth by targeting 
FBXW7. Zhou et al (37) also reported that miR-92a was upregulated in cervical cancer, and promoted cell proliferation and invasion by targeting FBXW7. Yang et al (38) reported that miR-92a promoted the tumor growth of HCC by targeting FBXW7. However, to the best of our knowledge, whether FBXW7 is a functional target of miR-92a in NSCLC cells has not been elucidated. In the present study, FBXW7 was validated as a target gene of miR-92a in NSCLC cells. In addition, the expression levels of FBXW7 in NSCLC tissues were low, and negatively correlated with miR-92a expression. Moreover, knockdown of FBXW7 in NSCLC cells attenuated the miR-92a inhibitor-induced reduction in cell viability. This indicated that an miR-92a inhibitor might inhibit NSCLC cell viability by targeting FBXW7.

In conclusion, in the present study, miR-92a was upregulated in NSCLC tissues and cell lines. Notably, miR-92a inhibition suppressed cell viability and promoted cell apoptosis by increasing FBXW7 expression in NSCLC cells. As a result, reducing miR-92a expression could have important implications for clinical management of NSCLC.

There are some limitations to the present study. For example, the clinical sample size was relatively small, and a larger sample size should be used in future studies to validate the accuracy of miR-92a as a biomarker for NSCLC. Furthermore, additional experiments in vivo should be performed to confirm whether miR-92a may be utilized as a therapeutic target for NSCLC. It has been shown that several targets can also be controlled by miR-92a in NSCLC, such as PTEN and double-strand-break repair protein rad21 homolog $1(31,39)$, which remains to be determined in future studies. Thus, further research is required to gain deeper insight into these mechanisms.

\section{Acknowledgements}

Not applicable.

\section{Funding}

No funding was received.

\section{Availability of data and materials}

All data generated or analyzed during this study are included in this published article.

\section{Authors' contributions}

DN, JT, YC, ZZ and BZ performed the experiments, contributed to data analysis and wrote the paper. ZY conceptualized the study design, contributed to data analysis and experimental materials. All authors read and approved the final manuscript.

\section{Ethics approval and consent to participate}

All individuals provided written, informed consent for the use of human specimens for clinical research. The present study was approved by Shanghai Ninth People's Hospital, Shanghai JiaoTong University School of Medicine.

\section{Patient consent for publication}

Not applicable.

\section{Competing interests}

The authors declare that they have no competing interests.

\section{References}

1. Siegel R, Naishadham D and Jemal A: Cancer statistics, 2013. CA Cancer J Clin 63: 11-30, 2013.

2. Torre LA, Bray F, Siegel RL, Ferlay J, Lortet-Tieulent J and Jemal A: Global cancer statistics, 2012. CA Cancer J Clin 65: 87-108, 2015.

3. Minna JD, Roth JA and Gazdar AF: Focus on lung cancer. Cancer Cell 1: 49-52, 2002.

4. International Early Lung Cancer Action Program Investigators, Henschke CI, Yankelevitz DF, Libby DM, Pasmantier MW, Smith JP and Miettinen OS: Survival of patients with stage I lung cancer detected on CT screening. N Engl J Med 355: 1763-1771, 2006.

5. Ambros V: The functions of animal microRNAs. Nature 431: 350-355, 2004.

6. Chen CZ: MicroRNAs as oncogenes and tumor suppressors N Engl J Med 353: 1768-1771, 2005.

7. Ma Y, Pan X, Xu P, Mi Y, Wang W, Wu X, He Q, Liu X, Tang W and An HX: Plasma microRNA alterations between EGFR-activating mutational NSCLC patients with and without primary resistance to TKI. Oncotarget 8: 88529-88536, 2017.

8. Krentz Gober M, Collard JP, Thompson K and Black EP: A microRNA signature of response to erlotinib is descriptive of TGF $\beta$ behaviour in NSCLC. Sci Rep 7: 4202, 2017.

9. Cui Y, Zhao L, Zhao S, Guo T, Li F, Li Z, Fang L, Wu T and Gu C: MicroRNA-30e inhibits proliferation and invasion of non-small cell lung cancer via targeting SOX9. Hum Cell 32: 326-333, 2019.

10. Liu F, Cai Y, Rong X, Chen J, Zheng D, Chen L, Zhang J, Luo R, Zhao $\mathrm{P}$ and Ruan J: miR-661 promotes tumor invasion and metastasis by directly inhibiting RB1 in non small cell lung cancer. Mol Cancer 16: 122, 2017.

11. Liu PJ, Ye YX, Wang YX, Du JX, Pan YH and Fang XB: miRNA-92a promotes cell proliferation and invasion through binding to KLF4 in glioma. Eur Rev Med Pharmacol Sci 23: 6612-6620, 2019.

12. Yu H, Song H, Liu L, Hu S, Liao Y, Li G, Xiao X, Chen X and He S: miR-92a modulates proliferation, apoptosis, migration, and invasion of osteosarcoma cell lines by targeting Dickkopf-related protein 3. Biosci Rep 39: BSR20190410, 2019.

13. Shigoka M, Tsuchida A, Matsudo T, Nagakawa Y, Saito $H$, Suzuki Y, Aoki T, Murakami Y, Toyoda H, Kumada T, et al: Deregulation of miR-92a expression is implicated in hepatocellular carcinoma development. Pathol Int 60: 351-357, 2010.

14. Chen E, Li Q, Wang H, Yang F, Min L and Yang J: miR-92a promotes tumorigenesis of colorectal cancer, a transcriptomic and functional based study. Biomed Pharmacother 106: 1370-1377, 2018

15. Jiang X, Li X, Wu F, Gao H, Wang G, Zheng H, Wang H, Li J and Chen C: Overexpression of miR-92a promotes the tumor growth of osteosarcoma by suppressing F-box and WD repeat-containing protein 7. Gene 606: 10-16, 2017.

16. Zhu Q, Zang Q and Jiang ZM: Enhanced expression of non coding miR 92a expression is implicated in the development of lung cancer. Eur Rev Med Pharmacol Sci 22: 1028-1034, 2018.

17. Livak KJ and Schmittgen TD: Analysis of relative gene expression data using real-time quantitative PCR and the 2(-Delta Delta C(T)) method. Methods 25: 402-408, 2001.

18. Yu Y, Zuo J, Tan Q, Zar Thin K, Li P, Zhu M, Yu M, Fu Z, Liang $\mathrm{C}$ and Tu J: Plasma miR-92a-2 as a biomarker for small cell lung cancer. Cancer Biomark 18: 319-327, 2017.

19. Coşarcă AS, Mocan SL, Păcurar M, Fülöp E and Ormenişan A: The evaluation of Ki67, p53, MCM3 and PCNA immunoexpressions at the level of the dental follicle of impacted teeth, dentigerous cysts and keratocystic odontogenic tumors. Rom J Morphol Embryol 57: 407-412, 2016.

20. Kent OA and Mendell JT: A small piece in the cancer puzzle: microRNAs as tumor suppressors and oncogenes. Oncogene 25: 6188-6196, 2006. 
21. Kong YW, Ferland-McCollough D, Jackson TJ and Bushell M: microRNAs in cancer management. Lancet Oncol 13: e249-e258, 2012.

22. Nasser MW, Datta J, Nuovo G, Kutay H, Motiwala T, Majumder S, Wang B, Suster S, Jacob ST and Ghoshal K: Down-regulation of micro-RNA-1 (miR-1) in lung cancer. Suppression of tumorigenic property of lung cancer cells and their sensitization to doxorubicin-induced apoptosis by miR-1. J Biol Chem 283 : 33394-33405, 2008.

23. Takamizawa J, Konishi H, Yanagisawa K, Tomida S, Osada H, Endoh H, Harano T, Yatabe Y, Nagino M, Nimura Y, et al: Reduced expression of the let-7 microRNAs in human lung cancers in association with shortened postoperative survival. Cancer Res 64: 3753-3756, 2004.

24. Yang Y, Ding L, Hu Q, Xia J, Sun J, Wang X, Xiong H, Gurbani D, Li L, Liu Y and Liu A: MicroRNA-218 functions as a tumor suppressor in lung cancer by targeting IL-6/STAT3 and negatively correlates with poor prognosis. Mol Cancer 16: 141, 2017.

25. Zhuang Z, Sun C and Gong H: High serum miR-484 expression is associated with the diagnosis and prognosis of patients with non-small cell lung cancer. Exp Ther Med 18: 4095-4102, 2019.

26. Li M, Guan X, Sun Y, Mi J, Shu X, Liu F and Li C: miR-92a family and their target genes in tumorigenesis and metastasis. Exp Cell Res 323: 1-6, 2014.

27. Guo J, Wen N, Yang S, Guan X and Cang S: miR-92a regulates oral squamous cell carcinoma (OSCC) cell growth by targeting FOXP1 expression. Biomed Pharmacother 104: 77-86, 2018.

28. Ke TW, Wei PL, Yeh KT, Chen WT and Cheng YW: miR-92a promotes cell metastasis of colorectal cancer through PTEN-mediated PI3K/AKT pathway. Ann Surg Oncol 22: 2649-2655, 2015

29. Chen ZL, Zhao XH, Wang JW, Li BZ, Wang Z, Sun J, Tan FW, Ding DP, Xu XH, Zhou F, et al: microRNA-92a promotes lymph node metastasis of human esophageal squamous cell carcinoma via E-cadherin. J Biol Chem 286: 10725-10734, 2011.

30. Bae S, Lee EM, Cha HJ, Kim K, Yoon Y, Lee H, Kim J, Kim YJ, Lee HG, Jeung HK, et al: Resveratrol alters microRNA expression profiles in A549 human non-small cell lung cancer cells. Mol Cells 32: 243-249, 2011.
31. Zhao J, Fu W, Liao H, Dai L, Jiang Z, Pan Y, Huang H, Mo Y, Li S, Yang G and Yin J: The regulatory and predictive functions of miR-17 and miR-92 families on cisplatin resistance of non-small cell lung cancer. BMC Cancer 15: 731, 2015.

32. Lu C, Shan Z, Hong J and Yang L: MicroRNA-92a promotes epithelial-mesenchymal transition through activation of $\mathrm{PTEN} / \mathrm{PI} 3 \mathrm{~K} / \mathrm{AKT}$ signaling pathway in non-small cell lung cancer metastasis. Int J Oncol 51: 235-244, 2017.

33. Welcker M and Clurman BE: FBW7 ubiquitin ligase: A tumour suppressor at the crossroads of cell division, growth and differentiation. Nat Rev Cancer 8: 83-93, 2008.

34. Minella AC and Clurman BE: Mechanisms of tumor suppression by the $\mathrm{SCF}$ (Fbw7). Cell Cycle 4: 1356-1359, 2005.

35. Li Z, Xiao J, Hu K, Wang G, Li M, Zhang J and Cheng G: FBXW7 acts as an independent prognostic marker and inhibits tumor growth in human osteosarcoma. Int J Mol Sci 16: 2294-2306, 2015.

36. Xiao Y, Yin C, Wang Y, Lv H, Wang W, Huang Y, Perez-Losada J, Snijders AM, Mao JH and Zhang P: FBXW7 deletion contributes to lung tumor development and confers resistance to gefitinib therapy. Mol Oncol 12: 883-895, 2018.

37. Zhou C, Shen L, Mao L, Wang B, Li Y and Yu H: miR-92a is upregulated in cervical cancer and promotes cell proliferation and invasion by targeting FBXW7. Biochem Biophys Res Commun 458: 63-69, 2015.

38. Yang W, Dou C, Wang Y, Jia Y, Li C, Zheng X and Tu K: MicroRNA-92a contributes to tumor growth of human hepatocellular carcinoma by targeting FBXW7. Oncol Rep 34: 2576-2584, 2015.

39. Ren P, Gong F, Zhang Y, Jiang J and Zhang H: MicroRNA-92a promotes growth, metastasis, and chemoresistance in non-small cell lung cancer cells by targeting PTEN. Tumour Biol 37: 3215-3225, 2016.

(i) $($ This work is licensed under a Creative Commons Attribution-NonCommercial-NoDerivatives 4.0 International (CC BY-NC-ND 4.0) License. 